\section{REPRÉSENTATIONS SOCIALES DE LA PRATIQUE ROCK EN MILIEU LYCÉEN}

\author{
par Jean-Marie SECA
}

On trouvera, dans cet article, les résultats d'une enquête par questionnaires, effectuée auprès de 281 lycéens parisiens de 15 à 18 ans. Après avoir insisté sur la difficulté pratique et théorique de construction d'indices adéquats et fidèles de questionnement sur l'influence effective du rock, il est précisé quel est le modèle de référence - en l'occurrence, celui de la culture de masse comme espace d'influences sociales servant à l'analyse des données recueillies. Les résultats permettent de vérifier l'existence d'une représentation sociale conférant au rock un caractère d'art potentiellement accessible à tous. La pratique rock en groupe est assez minoritaire ches les lycéens mème si l'influence de la musique comme mode de vie et attitude créative reste très appréciable. Enfin, le statut de minorité des groupes de rock est une variable expliquant le rejet ou la valorisation de ce courant auprès des sujets.

L'engouement pour la pratique rock ne date pas d'aujourd'hui en France comme ailleurs. L'émergence de minorités rock dans un contexte dominé par la culture de masse et le vedettariat apparaît, cependant, comme un fait nouveau dans le champ social de la France d'aujourd'hui (Seca, 1991, 1988, 1987 ; Daphy, 1988 ; Perriault et al. 1985; Hersent, 1988) même si la démocratisation musicale a commencé dès les années 50 par la multiplication puis le reflux, durant les années 60 , des sociétés orphéoniques (Gerbod, 1988).

Seront examinés et analysés dans les pages suivantes, la nature et le degré d'attirance des lycéens pour la pratique du rock (1).

Chercher à évaluer l'audience d'un phénomène culturel n'est pas chose aisée (2). Nous avons construit des catégories de classification des goûts musicaux, analysé le rapport des lycéens à l'avenir, leurs projets professionnels et personnels, leur vision de la réussite, leurs loisirs et centres d'intérêts. Nos résultats concernant ces dimensions n'ont pas semblé sensiblement différents par rapport à ceux commentés et construits par Boyer, Delcaux, Bounoure (1987), Green (1988), Mignon, Daphy et Boyer (1986), Boyer (1988) dans leurs propres enquêtes en milieu lycéen ou par rapport à d'autres enquêtes (Ministère de la Culture (1982), Guide de l'Étudiant (1978)), moins fouillées concernant ce domaine. A noter, cependant: nous constatons qu'au moins $63 \%$ des lycéens interrogés par nous sont allés au moins une fois à un concert de musique $(27,4 \%$ des lycéens, soit tous les mois, soit tous les trimestres). Ce taux assez élevé par rapport à la population lycéenne française (Boyer, Delcaux, Bounoure, 1987) indique que notre travail porte sur une catégorie de lycéens très insérés dans l'urbanité, sortant beaucoup et très influencés par la culture de masse. Notre analyse sera donc centrée essentiellement sur cette frange parisienne et urbaine des lycéens. Nous constatons, enfin, comme les chercheurs susmentionnés, l'existence, chez ce groupe social :

- d'une culture assez éclectique et diversifiée même si essentiellement référée à la musique commerciale et électronique ;

- et d'une anxiété à peine ironisée concernant leur avenir professionnel et familial.

Les filles sont, dans notre enquête aussi, un peu plus sensibles à l'art, plus motivées par l'investissement professionnel et affectif et beaucoup plus nuancées, sinon moins réceptives, quant à l'affiliation revendiquée, ouverte à un style rock pointu.

Avant de nous plonger dans l'analyse des résultats de notre étude, il est éclairant de faire un bref rappel du contexte théorique de l'objet social dont nous parlons.

\section{I. - DU ROCK "MAUVAIS GARGON" À L'ANALYSE dE SON INFLUENCE}

II est difficile d'être bref et synthétique concernant les thèmes de la culture "jeune", de "masse ", des phénomènes "de média", notions floues dont on entoure l'analyse du rock comme phénomène social. 
Le rock a souvent été associé à une vision subculturelle des groupes qui se constituaient en réseaux d'affinités autour de ses représentations sociales (3). Certains (Yonnet, 1985) en font, dans la même perspective, un élèment central de la compréhension de la société adolescente et tendent, sans l'expliciter, à adopter un point de vue fonctionnaliste sur le problème.

D'autres, enfin, soulignent la spécificité de son mode de production sociale, économique (Hennion, Vignolle 1978, Hennion 1980, 1983, Vignolle, 1980). Plus généralement, on remarque que beaucoup d'auteurs énoncent un lien assez fort et déterminant entre la naissance d'une certaine représentation du groupe " jeunes " et l'avènement d'une culture qui lui serait propre (Galland, 1984) et renverrait, ultérieurement et complémentairement, à une dimension essentielle du mode de vie de masse (Buxton, 1985, Mc Luhan, 1970, 1977, Altheide, 1976). La musique et les nouvelles technologies de communication sont alors conçues comme des pièces maîtresses d'une culture de masse qui baigne, en dernière instance, dans sa zone mentale et technique, tous les groupes sociaux.

La culture de masse est, alors, schématisée par la métaphore du fluide, du courant électrique dont les grands médias sont la matérialisation exacte. Morin (1966) parle, par exemple, d'un homme "ímbibé de culture urbaine " en décrivant des adolescents d'une petite ville de Province, porteurs de conduites novatrices par rapport à leur espace traditionnel de vie. Cette image de l'homme imbibé de culture-de-masse-urbaine, fait, alors, pendant à celle de l'homme inhibé en vogue à la fin du $19^{\circ}$ siècle.

C'est dans ce modèle théorique décrivant l'influence réciproque entre le groupe «jeunes" (en tant qu'espace investi imaginairement par les adultes autant que par les jeunes eux-mêmes) et le groupe "adultes" et où la culture de masse médiatise et fait circuler (en aplanissant les angles trop acérés des particularités) les représentations sociales du plaisir et de l'art, que nous situons (4) notre analyse du rock et de son audience (Seca, 1988).

Le plaisir musical, la transe deviennent alors des conduites sociales générales, d'ordre anthropologique. L'intériorisation d'un sens, d'une esthétique rock sont, selon nous à replacer autant dans une vision évolutive de l'environnement, dans un contexte essentiellement pluriel, métissé culturellement que dans une logique d'analyse faisant place à certaines théories globales sur la socialisation, l'art et la transe. Ce sont, cependant, des mouvements de synthèse et de disjonction culturelles qui caractérisent le rock et les représentations sociales qui le structurent. Mignon (1988, p. 32) semble d'accord avec nous quand il écrit: " on privilégiera une approche du "rock" comme produit de l'activité sociale, comme lieu de production de valeurs esthétiques et éthiques et comme lieu de conflits".
C'est cette référence au champ social et culturel, dans une relation dynamique et dialectique, qui va permettre au sens d'une pratique culturelle d'émerger aux yeux des divers acteurs qui la constituent, l'ignorent ou la critiquent. L'adhésion à un art ou à un courant obéit, aussi et surtout, à une logique de distinction sociale. Bourdieu indiquait, il y a quelques années (1980, p. 155): " il n'y a rien qui, autant que les goûts en musique, permette d'affirmer sa classe, rien aussi par quoi on soit infailliblement classé ".

Certaines recherches en psychologie sociale tendent à montrer (Tajfel, 1978) que l'attribution d'un trait, d'une signification à une forme dépend aussi des sens qu'elle prend dans les groupes opposés à d'autres groupes, dans l'interaction elle-même.

Enfin, l'influence dite "indirecte "n'est pas sans effet sur la constitution et l'évolution des goûts musicaux (5).

Tous ces points incitent à penser le rock et les engouements qui l'accompagnent, comme l'effet (et espace de médiation) d'une action à distance entre le social et l'individu.

L'analyse de la création culturelle en termes de constitutions de réseaux d'affinité et de groupes (Johnstone et Katz, 1957) est à resituer dans ce contexte.

\section{II. - MODĖLES ROCK: DE L'AMOUR DU ROCK À SA PRATIQUE}

Notre approche dans cette enquête prend en compte le côté "aérien", donc insaisissable, non figé du rock (6). Une lecture en termes de représentations sociales (Moscovici 1976), statistiquement spécifiée et sériée dans des opinions-items sur les groupes de rock serviront de supports méthodologiques à notre travail. C'est, en effet, à travers des évaluations, faites par les lycéens, concernant des items pré-construits et quelques questions ouvertes que nous explorerons les représentations sociales de la pratique rock.

Il apparaît, d'abord, qu'une relative faiblesse de l'activité musicale rock confère à l'univers culturel lycéen un caractère essentiellement mass-médiatique, même si la pratique musicale, en général, attire.

Le lycéen reste avant tout un auditeur-consommateur. Voici les résultats que nous avons trouvés à ce propos:

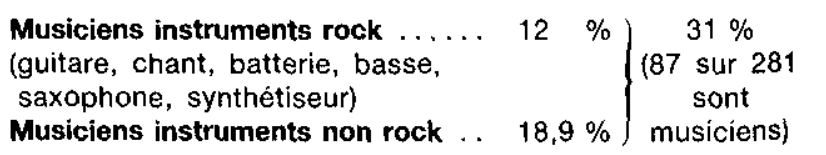

Lycéens non musiciens ........ $69 \%$ (194/281) 
Nous avons, donc, sept lycéens sur dix environ qui ne sont pas musiciens et un sur dix qui joue un instrument rock (par référence à une analyse des instruments les plus pratiqués dans les groupes de rock amateurs (SECA 1988, ch. 3)).

A ceux qui ne font pas de musique, nous avons demandé s'ils désiraient en faire dans un futur plus ou moins éloigné. Voici les résultats :

- ne désirant pas en faire:

- 80 sur $194=41,2 \%(28,5 \%$ de l'ensemble $)$

- désirant en faire :

- 114 sur $194=58,8 \%$ (40,5\% de l'ensemble)

L'audience du modèle musical, au sens large, est, donc, assez importante : si l'on ajoute à ceux qui désirent faire de la musique ceux qui en font effectivement, on obtient le chiffre de $71,5 \%$ (201 sur 281 élèves).

\section{Attirance pour la pratique rock elle-même}

La musique attire les lycéens. Cela ne signifie pas, pour autant, une volonté égale d'entrer dans un groupe de rock:

- ne désirant pas faire partie d'un

groupe rock ................

- désirant appartenir à un groupe

$78,3 \% \quad(220 / 281)$

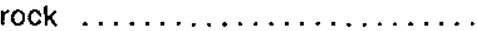

$19,6 \%$

$2,1 \%$

Nous remarquons l'existence d'une relative gradation dans cet attrait pour la pratique rock selon un critère renvoyant à la perception positive de la pratique musicale en général.

\begin{tabular}{|c|c|c|c|}
\hline $\begin{array}{l}\text { Effectif } \\
\% \text { colonne } \\
\end{array}$ & $\begin{array}{c}\text { Désir (-) de } \\
\text { faire partie } \\
\text { d'un groupe } \\
\text { rock }\end{array}$ & $\begin{array}{c}\text { Désir (t) de } \\
\text { faire partie } \\
\text { d'un groupe } \\
\text { rock }\end{array}$ & Ensemble (") \\
\hline $\begin{array}{l}\text { Désir }(-) \text { de faire de la } \\
\text { musique }\end{array}$ & $\begin{array}{l}34,55^{76} \\
95,00\end{array}$ & $7,27^{4} 5,00$ & $29,09^{80} 100$ \\
\hline $\begin{array}{l}\text { Désir }(+) \text { de faire de la } \\
\text { musiqué }\end{array}$ & $37,73^{83}$ & $\begin{array}{l}{ }_{54,55}^{30} \\
\\
26,55\end{array}$ & 41,09 \\
\hline $\begin{array}{l}\text { Non réponses } \\
= \\
\text { musiciens }\end{array}$ & $\begin{array}{c}61 \\
27,72 \\
\end{array} 74,39$ & ${ }_{38,18}^{21} 25,61$ & $29,82^{82} 100$ \\
\hline Ensemble (*) & $100^{220} 80,00$ & $100^{55} 20,00$ & $100^{275} 100$ \\
\hline
\end{tabular}

(7) Moins les non-réponses ( $=6$ individus).

Test du CHI-DEUX:CHI-OEUX $=15,9 ; \mathrm{DDL}=2 ; \mathrm{p}=0,0003$.
Les non-musiciens attirés par la pratique musicale sont, proportionnellement, plus nombreux que les musiciens eux-mêmes, à vouloir faire partie d'un groupe de rock. C'est là une caractéristique importante des représentations sociales de la pratique musicale rock: son apparence d'accessibilité.

On note, d'ailleurs, que 10 musiciens sur 87 (soit $11,5 \%$ ) appartiennent à des formations musicales autres que rock et que 3 seulement (sur ces mêmes 87 ) sont membres d'un groupe de rock (soit 3,4\%).

Les musiciens, réellement engagés dans un groupe sont donc très minoritaires ( $=13$ en tout, soit $14,9 \%$ des musiciens et $4,6 \%$ de l'ensemble des lycéens).

Faire du rock en groupe et faire de la musique tout seul n'impliquent évidemment pas les mêmes projets (création d'un répertoire, d'une image sociale, innovation collective, recherche d'un minimum de vedettariat, investissements temporels et financiers).

Une interprétation de ces résultats est difficile. Ils suggèrent, en effet, l'idée que $95,4 \%$ des lycéens interrogés sont de simples auditeurs ou des musiciens soljtaires, en chambre, apprentis-dilettante. Mais, on peut aussi inverser le problème et compléter l'analyse, en soulignant à quel point le mode de vie musical a d'influence dans l'attrait qu'il exerce non seulement en tant que culture consommée mais aussi en tant que culture créée, auto-produite $(=7$ lycéens sur 10 sont musiciens ou ont envie de le devenir).

Les raisons avancées par ceux qui ne désirent pas appartenir à un groupe de rock nous renseignent un peu sur l'attitude de l'ensemble des lyceens ne faisant pas partie d'une formation musicale. Ils avancent les raisons suivantes :

- le rock n'est pas une musique qui attire, qui plaît ....... 10,7\%

- la musique n'est pas un centre d'intérêt ............ 20,6\%

- il existe d'autres passions 6,8\% d'attrait rée

$38,1 \%$ pour la musique et le rock

- Ia préférence est donnée à Affirmation l'écoute, au statut d'auditeur $7,1 \%$

- le sujet se sent peu doué pour la musique .......... 14,6\%

- faire du rock, c'est trop difficile pour y arriver ........ 11,4\%

$33,1 \%$ de ne pas implicite du côté des créateurs

- Non-réponses ............ 28,8\%

Les raisons avancées ici oscillent entre l'affirmation d'un sentiment d'impuissance et celle du rejet d'un modèle de culture vu comme éloigné de soi. Le sentiment d'impuissance consiste à affirmer que l'on ne peut ou que 
I'on ne voit pas comment on pourrait être de l'autre côté de la barrière. Ce sont, bien entendu, les plaisirs multiples de la situation d'auditeurs qui sont avancés comme explication plausible de ce sentiment.

Au-delà du manque d'intérêt pour la musique ou le rock, nous sommes ici en face de l'expression d'une opinion assez ancrée chez les auditeurs selon laquelle la musique serait une affaire de professionnels et le vedettariat une sphère sociale lointaine, olympienne.

C'est d'ailleurs contre ce sentiment-là que les premiers groupes punks, consommateurs nauséeux de rock, se sont insurgés avec une violence dont les rituels sont restés célèbres (Seca, 1988).

\section{L'envie de la pratique rock vient en rockant}

Le monde des petits groupes de rock n'est pas un milieu complètement étranger aux lycéens : $35,2 \%$ affirment connaître personnellement un ou plusieurs groupes (7). Des liens existent, donc, avec ceux que nous qualifions de minorités rock. II semble, cependant, que l'examen de certaines données nous permette de confirmer l'hypothèse suivante: plus le milieu rock au sens large est directement approché et plus celui-ci exercera un attrait sur le spectateur (8) dans son envie de pratiquer, de faire partie d'un groupe:

a. Plus souvent on fréquentera les salles de concert, et plus on aura envie de faire partie d'un groupe de rock.

b. La plus grande fréquentation des salles de concert, de leur ambiance, a plus d'effets sur le désir d'appartenir à un groupe de rock que sur celuj de faire de la musique.

Ceux qui ne fréquentent jamais les salles de concert sont $83,2 \%$ à ne pas désirer faire partie d'un groupe de rock (9).

c. Le fait d'avoir un ou plusieurs groupe (s) de rock dans ses relations personnelles est un autre élément influençant les vocations rock et ceci d'autant plus que le nombre de groupes connus est important.

Si l'on fait la somme de l'ensemble des individus entretenant des relations personnelles avec un ou plusieurs groupe (s) de rock, on note que $32,6 \%$ d'entre eux désirent appartenir à un groupe alors que seuls $13,7 \%$ ne connaissant pas de groupes affirment un tel désir (10).

d. Plus on a approché le milieu rock, et plus l'envie de participer à un groupe est importante. Cela se vérifie a fortiori, lorsque l'on analyse l'attitude d'ex-membres de groupes de rock qui sont au nombre de 16 sur 281 $(5,8 \%)$. Leur désir de refaire partie d'un groupe sera, là aussi, très élevé (11).
L'appartenance passée à un groupe de rock semble avoir provoqué une passion durable pour la musique au sens large. On remarque, en effet, que tous les ex-membres de groupes de rock désirent faire de la musique dans l'avenir.

Notons que, du même coup, certains ex-membres de groupes ne se considèrent pas comme des musiciens. D'où la particularité de 7 ex-membres de groupes ne faisant pas de la musique (à leurs dires) mais désirant en faire dans le futur. Là aussi, la spécificité de l'engagement dans un groupe de rock réside dans le fait que l'on ne se sent pas paralysé par le manque de maîtrise technique musicale: le but est l'expression de soi.

e. De même, on peut remarquer une plus forte tendance à vouloir faire partie d'un groupe de rock chez les amateurs de pop-rock, les hard-rockers, et ceux que nous nommons les éclectiques (12). Les amateurs de hard-rock sont les plus attirés par le modèle du " guitarhero " et ce n'est pas un hasard s'ils affirment avec une grande unanimité le désir de fonder un groupe de rock dans l'avenir.

On peut donc admettre, sur la base des données cidessus, que les lycéens plus familiarisés avec le milieu des concerts et des musiciens sont largement plus favorables au modèle rock. L'attirance pour le rock semble, aussi, se manifester à la suite des spectacles et des multiples auditions de disques très orientés vers ce courant musical.

Le manque d'attrait pour le modèle expressif rock apparaît donc comme étant, à la fois, une méconnaissance et une imperméabilité volontaire à son influence, un désír d'affirmer autrement sa sensibilité. L'influence du mode de vie musical n'en reste pas moins forte pour beaucoup de lycéens. Mais ce que certains d'entre eux refusent dans les groupes de rock est le résultat d'une attribution d'un statut marginal et mythique à ce milieu. Examinons quelle est la prégnance d'une telle attribution.

\section{III. - INFLUENCE, MARgINALITÉ, PROXIMItÉ : REPRÉSENTATIONS SOCIALES DES GROUPES DE ROCK ET DE LEUR ACTION}

Nous avons demandé aux lycéens d'évaluer un certain nombre d'items attribuant des traits psychologiques et sociaux à la pratique et au milieu rock (13). Tous les lycéens citent des musiciens rock parallèlement à leurs autres préférences musicales. Une extrême minorité y est très réticente (2 à $3 \%$ d'après les analyses de correspondances et une classification ascendante hiérarchique consécutive). Ceci étant posé au départ, examinons quelle est la nature de l'image des groupes de rock chez les lycéens selon trois dimensions : 
1) identification,

2) marginalité positivement perçue et négativement perçue,

3) appréciation de l'influence du rock sur la société.

\subsection{Identification}

II nous est difficile de savoir, à partir de nos données, à quel degré une identification existe chez les lycéens envers les groupes de rock. Là aussi, nous demeurons au niveau des représentations sociales et des attributions de traits renvoyant à une proximité psychique de ces meneurs musicaux des médias avec les sujets interrogés.

\begin{tabular}{|c|c|c|c|c|}
\hline & $\begin{array}{c}\text { Note } \\
\text { (moyenne) } \\
\text { Max. : } 6 \\
\text { Min. : } 0\end{array}$ & $\begin{array}{c}\text { Individus } \\
\text { nepondant } \\
\text { positivt. } \\
(+1+3)\end{array}$ & $\begin{array}{l}\text { Individus } \\
\text { repondamt } \\
\text { négativt. } \\
(-1+\mathbf{a}-3)\end{array}$ & $\begin{array}{c}\text { Mon } \\
\text { réponses }\end{array}$ \\
\hline $\begin{array}{l}\text { - les groupes de rack } \\
\text { sont proches de vos } \\
\text { manieres de vivre ... }\end{array}$ & 3,1 & $\begin{array}{r}135 \\
(48 \%)\end{array}$ & $\begin{array}{r}146 \\
(52 \%)\end{array}$ & 0 \\
\hline $\begin{array}{l}\text { - les membres des mi- } \\
\text { norités rock ont les } \\
\text { mêmes problèmes } \\
\text { que vous ........... }\end{array}$ & 4,9 & $\begin{array}{c}233 \\
(82,9 \%)\end{array}$ & $\begin{array}{c}42 \\
(14,9 \%)\end{array}$ & $\begin{array}{c}6 \\
(2,2 \%)\end{array}$ \\
\hline $\begin{array}{l}\text { - les membres des mi- } \\
\text { norités sont des gens } \\
\text { comme tout le monde }\end{array}$ & 4,8 & $\begin{array}{c}228 \\
(81,1 \%)\end{array}$ & $\begin{array}{c}48 \\
(17,1 \%)\end{array}$ & $\begin{array}{c}5 \\
(1,8 \%)\end{array}$ \\
\hline $\begin{array}{l}\text { - les groupes de rock } \\
\text { sont attirants ........ }\end{array}$ & 4,4 & $\begin{array}{c}219 \\
(77,9 \%)\end{array}$ & $\begin{array}{c}58 \\
(20,7 \%)\end{array}$ & $\begin{array}{c}4 \\
(1,4 \%)\end{array}$ \\
\hline $\begin{array}{l}\text { - faire du rock est un } \\
\text { loisir comme un autre }\end{array}$ & 3,8 & $\begin{array}{c}161 \\
(57,3 \%)\end{array}$ & $\begin{array}{r}118 \\
(42 \%)\end{array}$ & $\stackrel{2}{2} \%$ \\
\hline
\end{tabular}

\subsection{Marginalité positive et marginalité négative}

L'attribution d'un statut minoritaire à un groupe ou à un individu est un processus qui est basé sur la perception ambiguë qu'en ont les sujets évaluateurs. Nous avons vu que les groupes de rock sont considérés comme attirants pour une très grande partie des lycéens même si les avis sont plus nuancés pour approuver l'idée d'une proximité au niveau du mode de vie.

Nous avons tenté de scinder les attributions d'étiquettes marginales en deux types (négatifs et positifs) pour comprendre comment elles s'articulaient les unes aux autres.
Voici les résultats concernant ces deux dimensions :

Marginalité négative

\begin{tabular}{|c|c|c|c|c|}
\hline $\begin{array}{l}\text { Les membres des } \\
\text { groupes de rock... }\end{array}$ & $\begin{array}{c}\text { Note } \\
\text { (moyenne) } \\
\text { Max.: } \mathbf{6} \\
\text { Min. : } 0\end{array}$ & $\begin{array}{c}\text { Individus } \\
\text { repondant } \\
\text { positivt. } \\
(+1 \mathrm{a}+3)\end{array}$ & $\begin{array}{c}\text { Indlvidus } \\
\text { répondant } \\
\text { neggativt. } \\
(-1 \mathrm{a}-3)\end{array}$ & $\begin{array}{l}\text { Non } \\
\text { repponses }\end{array}$ \\
\hline - sont sectaires ...... & 3 & $\begin{array}{c}119 \\
(42,3 \%)\end{array}$ & $\begin{array}{c}150 \\
(53,4 \%)\end{array}$ & $\begin{array}{c}12 \\
(4,3 \%)\end{array}$ \\
\hline $\begin{array}{c}\text { - sont d'éternels ado- } \\
\text { lescents .......... }\end{array}$ & 3,2 & $\begin{array}{c}126 \\
(44,8 \%)\end{array}$ & $\begin{array}{c}153 \\
(54,4 \%)\end{array}$ & $\begin{array}{c}2 \\
(0,7 \%)\end{array}$ \\
\hline - sont des marginaux.. & 2,9 & $\begin{array}{c}115 \\
(40,9 \%)\end{array}$ & $\begin{array}{r}163 \\
(58 \%)\end{array}$ & $\begin{array}{c}3 \\
(1,1 \%)\end{array}$ \\
\hline — sont des angoissés. & 2,2 & $\begin{array}{c}62 \\
(22,1 \%)\end{array}$ & $\begin{array}{c}215 \\
(76,5 \%)\end{array}$ & $\begin{array}{c}4 \\
(1,4 \%)\end{array}$ \\
\hline $\begin{array}{c}\text { - n'ont que ça comme } \\
\text { ambition ........... }\end{array}$ & 3,7 & $\begin{array}{r}160 \\
(57 \%)\end{array}$ & $\begin{array}{c}115 \\
\langle 40,9 \%\rangle\end{array}$ & $\begin{array}{c}6 \\
(2,1 \%)\end{array}$ \\
\hline — fuient la societé ..... & 3,2 & $\begin{array}{c}129 \\
(45,9 \%)\end{array}$ & $\begin{array}{c}152 \\
(54,1 \%)\end{array}$ & 0 \\
\hline
\end{tabular}

Marginalité positive

\begin{tabular}{|c|c|c|c|c|}
\hline $\begin{array}{l}\text { Les membres des } \\
\text { groupes de rock } \\
\text { sont... } \\
\text { Réponses }\end{array}$ & $\begin{array}{c}\text { Mote } \\
\text { (moyerine) } \\
\text { Max. }: 6 \\
\text { Min.: }: 0\end{array}$ & $\begin{array}{c}\text { Individus } \\
\text { répondant } \\
\text { positivt. } \\
(+1 \mathrm{a}+3) \\
\end{array}$ & $\begin{array}{c}\text { Isdividus } \\
\text { répondant } \\
\text { négativt. } \\
(-1 \mathrm{a}-3)\end{array}$ & $\begin{array}{l}\text { Non } \\
\text { reponses }\end{array}$ \\
\hline — des êtresá part..... & 2,8 & $\begin{array}{c}105 \\
(37,4 \%)\end{array}$ & $\begin{array}{c}174 \\
(61,9 \%)\end{array}$ & $(0,7 \%)$ \\
\hline $\begin{array}{l}- \text { des individus hors du } \\
\text { commun ............ }\end{array}$ & 3,1 & $\begin{array}{c}124 \\
\langle 44,1 \%)\end{array}$ & $\begin{array}{c}155 \\
(55,2 \%)\end{array}$ & $\begin{array}{l}2 \\
(0,7 \%)\end{array}$ \\
\hline $\begin{array}{l}\text { - plus actifs que la } \\
\text { moyenne............. }\end{array}$ & 3,6 & $\begin{array}{c}165 \\
(58,7 \%)\end{array}$ & $\begin{array}{c}115 \\
\langle 40,9 \%)\end{array}$ & $\begin{array}{c}1 \\
(0,4 \%)\end{array}$ \\
\hline $\begin{array}{c}\text { - plus créatifs que } 1 \text {. } \\
\text { moyenne........... }\end{array}$ & 3,8 & $\begin{array}{c}180 \\
(64,1 \%)\end{array}$ & $\begin{array}{c}101 \\
(35,9 \%)\end{array}$ & 0 \\
\hline $\begin{array}{l}\text { - ॥ faut avoir un don } \\
\text { pour la musique pour } \\
\text { faire partie d'un } \\
\text { groupe de rock ..... }\end{array}$ & 4,2 & $\begin{array}{c}205 \\
(72,9 \%)\end{array}$ & $\begin{array}{r}73 \\
(26 \%)\end{array}$ & $\begin{array}{c}3 \\
(1,1 \%)\end{array}$ \\
\hline
\end{tabular}

Les lycèens ne cherchent apparemment pas à étiqueter les membres des groupes de rock comme des exclus et des déviants. L'analyse des correspondances révèle aussi que les sujets trouvant les rockers sectaires, peu doués, angoissés, marginaux, éternels adolescents sont 
généralement assez peu attirés par le rock et le modèle expressif qui lui est propre. A un second degré, l'attribution de marginalité, même si elle apparaît négative, est une étiquette valorisée et côtoie les qualificatifs plus valorisants socialement (plus actif, créatif...). Ce changement de valeur des traits de marginalité est particulièrement décelable pour des termes comme "éternels adolescents ", "marginaux", "fuyant la société" et même " angoissés". Nous ne pouvons présenter toutes les données (analyses de correspondances) (14) concernant ces dimensions, mais il semble bien que, pour une partie des sujets, les traits décrivant le statut minoritaire, quels qu'ils soient, deviennent désirables et soient associés à une valorisation de la situation d'artiste (le mythe de l'artiste maudit).

Plus généralement, en prenant l'ensemble des sujets comme référence, on note une grande difficulté pour attribuer aux rockers des qualités de charisme ou des traits désignant une personnalité supérieure $(=$ "hors du commun ", "êtres à part "). Cette indécision est moins grande quand il s'agit de qualifier des conduites (= "actifs", "créatifs") plutôt que des traits de personnalité. D'un certain point de vue, les sujets ne veulent surtout pas attribuer un pouvoir supérieur ou établir une hiérarchisation entre les artistes musiciens et eux-mêmes. On remarquera aussi, malgré cela, que les sujets plus favorables au rock et désirant en faire se représenteront beaucoup plus les rockers comme des individus supérieurs. La fraternisation semble alors renfermer les germes d'une admiration, d'une soumission à des valeurs et à des hommes qui deviennent des points de comparaison avec soi.

Le seul point d'attribution positive sur lequel les lycéens semblent être assez d'accord, est celui du don et de la créativité. Même si, à un premier niveau, la nonattribution de don et de qualités supérieures est un moyen d'innoculer un jugement proche de l'indifférence ou de l'hostilité aux rockers, il semble que le fait d'être doué soit le seul point indépassable de l'activité musicale (rock ou pas). Le don vu comme étant de l'ordre du quasi-inné, du quasi-biologique, peut devenir une fonction, un appendice (comme le chant du rossignol) et être admis comme tel (c'est-à-dire naturel) sans que des attributions de traits socialement supérieurs soient faites en même temps ou ressenties comme associées à ce trait. De la même manière, le fait d'attribuer un trait de marginalité, même négativement perçu, peut être vu par certains sujets comme un moyen de donner une valeur (donc, les reconnaître) à des groupes qu'ils méprisent.

Les anti-rockers tendront donc, pour certains, à nier tout caractère distinctif aux musiciens de groupes rock en renvoyant ceux-ci dans la zone de l'ignorance, de l'inconnu et de l'indifférence, forme subtile de l'exclusion et de la dévalorisation.

\subsection{Appréciation de l'influence du rock sur la société}

Voici les résultats concernant cette dimension :

\begin{tabular}{|c|c|c|c|c|}
\hline Faire du rock... & $\begin{array}{c}\text { Note } \\
\text { (moyenne) } \\
\text { Max.: } 6 \\
\text { Min.: } 0\end{array}$ & $\begin{array}{l}\text { Individus } \\
\text { rópondant } \\
\text { positivt. } \\
(+1 \text { a }+3)\end{array}$ & $\begin{array}{c}\text { Individus } \\
\text { répondant } \\
\text { négativt. } \\
(-1 \mathrm{a}-3)\end{array}$ & $\begin{array}{c}\text { Non } \\
\text { réponses }\end{array}$ \\
\hline $\begin{array}{l}\text { - C'est faire quelque } \\
\text { chose pour étre re- } \\
\text { connu socialement .. }\end{array}$ & 2,6 & $\begin{array}{c}95 \\
(33,8 \%)\end{array}$ & $\begin{array}{c}181 \\
(64,4 \%)\end{array}$ & $\begin{array}{c}5 \\
(1,8 \%)\end{array}$ \\
\hline $\begin{array}{l}\text { - C'est avoir envie de } \\
\text { devenir une vedette.. }\end{array}$ & 3,7 & $\begin{array}{c}175 \\
(62,3 \%)\end{array}$ & $\begin{array}{r}104 \\
(37 \%)\end{array}$ & $\stackrel{2}{2} \%$ \\
\hline $\begin{array}{l}\text { - C'est avoir envie de } \\
\text { communiquer avec } \\
\text { les gens } \ldots \ldots \ldots \ldots\end{array}$ & 5 & $\begin{array}{c}252 \\
(89,7 \%)\end{array}$ & $\begin{array}{c}27 \\
(9,6 \%)\end{array}$ & $\begin{array}{c}2 \\
(0,7 \%)\end{array}$ \\
\hline $\begin{array}{l}\text { - C'est pour gagner de } \\
\text { l'argent agréablement }\end{array}$ & 3,9 & $\begin{array}{c}190 \\
(65,6 \%)\end{array}$ & $\begin{array}{c}89 \\
(31,7 \%)\end{array}$ & $\begin{array}{l}2 \\
(0,7 \%)\end{array}$ \\
\hline $\begin{array}{l}\text { - Les groupes de rock } \\
\text { ont une action sur les } \\
\text { mentalités .......... }\end{array}$ & 4,4 & $\begin{array}{r}225 \\
(80 \%)\end{array}$ & $\begin{array}{c}55 \\
(19,6 \%)\end{array}$ & $\stackrel{1}{(0,4 \%)}$ \\
\hline $\begin{array}{l}\text { - C'est une volonté de } \\
\text { délivrer un message . }\end{array}$ & 4,6 & $\begin{array}{c}240 \\
(85,4 \%)\end{array}$ & $\begin{array}{c}39 \\
(13,9 \%)\end{array}$ & $\begin{array}{l}2 \\
(0,7 \%)\end{array}$ \\
\hline $\begin{array}{c}\text { - Les groupes rock font } \\
\text { changer le monde ... }\end{array}$ & 2,8 & $\begin{array}{c}103 \\
(36,6 \%)\end{array}$ & $\begin{array}{c}175 \\
\{62,3 \%)\end{array}$ & $\begin{array}{l}3 \\
(1,1 \%)\end{array}$ \\
\hline $\begin{array}{c}\text { - C'est une manière de } \\
\text { se révolter contre } \\
\text { lordre établi } \ldots \ldots \ldots\end{array}$ & 3,5 & $\begin{array}{r}163 \\
(58 \%)\end{array}$ & $\begin{array}{c}116 \\
(41,3 \%)\end{array}$ & $\begin{array}{c}2 \\
(0,7 \%)\end{array}$ \\
\hline
\end{tabular}

L'engagement dans un groupe n'est pas perçu explicitement comme un besoin de reconnaissance sociale même si l'on soupçonne tout rocker de vouloir devenir une vedette. Entre la reconnaissance sociale et la consécration sociale, il semble exister une certaine différence de perception qu'on peut associer à l'enjeu, dont nous parlions plus haut, d'attribution aux rockers de qualités supérieures. Les effets de l'action rock sont appréciés avec une certaine prudence puisqu'on pensera que les groupes ont une action sur les mentalités mais qu'ils ne font pas vraiment changer le monde. L'influence rock est spirituelle. Elle est donc action sur le mental et transmission d'un contenu $(=$ le message sans qu'il y ait précision sur ce qu'il véhicule). De la même façon, les sujets seront plus d'accord pour évaluer l'activité rock comme une manière de se révolter contre l'ordre établi et, surtout, 
pour communiquer avec les gens. Cette conscience que la révolte est purement formelle est, d'ailleurs, associée à l'idée qu'elle peut s'accompagner d'avantages pécuniaires.

L'idée subsiste donc que le rock est un énorme moyen artistico-commercial pour faire passer des émotions, des contenus et pour communiquer au sens large avec les gens. Le statut du rocker demeure, cependant, marqué par une saveur d'exotisme que les lycéens, malgré leur volonté de proximité psychique avec lui (" ils sont comme tout le monde ") confirment dans leur évaluation de son statut de marginalité.

La vie d'artiste est chargée de mythes qui ont une incidence directe sur les représentations sociales qui lui sont attachées. Une identification existe, cependant, entre les lycéens et les rockers: elle semble plus refléter un langage de fan ou d'adorateur relativement critique que celui d'un futur musicien envieux. L'accent mis sur le plaisir, la communication, le message, les émotions, le défoulement, le don, la créativité plutôt que sur la reconnaissance sociale, la transformation du monde, l'individualisme, la moins grande valorisation de la vocation pour la création permettent, dans une certaine mesure, de comprendre l'identification des lycéens vis-à-vis des rockers comme fondée sur l'ambivalence et surtout un sentiment d'auditeur-consommateur de produits musicaux. La musique est avant tout un plaisir plus qu'une recherche intellectuelle même si elle agit sur les esprits.

\section{IV. - AUTO-ATTRIBUTION D'UN LOOK ET VISIBILITÉ SOCIALE}

L'influence de la musique est, donc, ressentie comme matérialisable et corporéisée.

$74,7 \%$ des lycéens estiment, en effet, qu'il y a un grand rapport entre la musique qu'on écoute et les vêtements qu'on porte. L'éveil de la sexualité chez les adolescents qu'ils sont, peut être associée chez les lycéens à l'excessive stéréotypie dont ils font preuve ici : "On est comme on paraît, on paraît comme on sent et l'on sent aujourd'hui l'environnement par les symboles musicaux". II semble que cette catégorisation (volontairement présentée comme générale) soit surtout une attribution d'ordre externe (vis-à-vis des autres).

La question plus centrée sur soi concernant la définition de son propre look (question ouverte) fait apparaître, à l'inverse, une très faible référenciation aux termes musicaux : $10,7 \%$ seulement d'auto-attributions musicales pour qualifier sa propre apparence vestimentaire.

En général, les lycéens cherchent à disparaître derrière une normativité rassurante ("simples, communs, normaux" [13,2\%], "bon chic-bon genre, conventionnels, élégants" $[18,1 \%]]$. Cette volonté de n'afficher aucun des signes de distinction qui stigmatisent ou désignent une originalité trop accentuée, trop exhibitionniste est une représentation à la mode depuis quelques années en Europe (Sommer et Wind [1986], Obalk, Soral et Pasche [1984]). C'est ce qu'on a appelé, communément, le "bécébégisme" dont la nature obsessionnelle (se dissimuler dans le normal avec une pointe de bon goût) apparaît dans le luxe de détails apporté chez le bcbg (bon chic bon genre) à l'autocomposition de son vêtement.

Au-delà des clichés marketing, que les termes comme bcbg véhiculent, nous pensons que cette volonté d'être normal, voire plus normal que les autres $(31,3 \%$ $(=13,2 \%$ de "normaux, communs $+18,1 \%$ de " bcbg ") de nos réponses à la question ouverte sur l'autodéfinition de soi) est un exemple de plus de ce que Codol (1979) a théorisé sous le phénomène dit " d'effet PIP " ou "conformité supérieure de soi ".

Cette forme de recherche de distinction se manifeste aussi par l'affirmation d'une attitude de non-comparaison culturelle ("être hors-mode, très personnel, composant soi-même son propre costume" $(=26,3 \%)$ des-réponses). Lemaine (1974) a lui aussi décrit par le menu, la spécificité de cette recherche de différenciation par la mise à l'écart de soi par rapport aux échelles communes d'évaluation. On peut d'ailleurs associer à cette tendance la minorité de lycéens $(5,7 \%)$ disant vouloir provoquer par l'habillement, être excentrique. S'ex-centrer, c'est en effet, se mettre hors de comparaison.

Enfin, nous avons noté l'émergence d'une dernière catégorie de réponses classées par nous, sous la catégorie "cool, relax" et renvoyant alors à l'affirmation par le mental de l'apparence extérieure. Quoi de moins variable, en effet, que l'affirmation d'un trait de personnalité, désignant, surtout, l'intérieur alors que la question posée portait sur le "look" (= apparence vestimentaire et corporelle). Cette catégorie de réponse est, de plus, inévitable puisque la représentation sociale mis en branle ici, ne peut que se situer à l'entrecroisement de l'affirmation du Soí et de l'affichage social de son corps. Les réponses "cool, relax $(21 \%)$ " renvoient au "Soi " psychique mais aussi à un ètat corporel plus que vestimentaire, à un désir de mise hors de comparaison sociale au même titre que chez les " hors mode $(26,3 \%)$ » ou les "excentriques $(5,7 \%)$ \%.

L'extrême diversité des auto-qualificatifs vestimentaires, même en ce qui concerne les auto-évaluations empreintant une terminologie musicale, rejoint les résultats de classifications des styles musicaux faites par nous (Seca, 1987) et par d'autres (Mignon, Daphy, Boyer, 1986). Le vêtement comme la consommation musicale 
sont rarement avoués comme des conduites d'imitation. L'influence des styles et des minorités se fait d'une façon indirecte (Moscovici, Mugny, 1988, Aebischer, Hewstone, Henderson, 1984).

Les lycéens ne sont pas dupes de l'existence de cette influence: ne soulignent-ils pas (à $74,7 \%$ ) l'existence d'un rapport entre musique qu'on écoute et vêtement qu'on porte? Cette conscience de subir une influence n'est pas verbalisée et désignée avec précision.

Indépendamment du manque évident de lien définitif entre histoire des rock et "grammaire" des look, les réponses trouvées par nous nous incitent à penser le vêtement comme un enjeu plus sensible et significatif du désir de rester original que la créativité musicale. Le vêtement plus accessible que la pratique artistique, est une réserve symbolique commode de signes malléables d'approbation sociale. II est plus soumis au regard majoritaire même si quelques élites ou minorités "bricolent " par son intermédiaire des univers dérangeants.

If y a d'ailleurs quelques liens (différences par rapport à la moyenne) entre le fait d'estimer un grand rapport entre vêtement et musique qu'on écoute et celui de ne pas être musicien et de vouloir le devenir. L'élévation du rapport estimé entre vêtement et musique est lié, aussi, au désir de faire partie d'un groupe de rock mais d'une façon moins affirmée (15).

Ces résultats indiquent qu'un lien existe entre désir de visibiljié sociale et volonté de créer par soi-même. Une fois ce désir satisfait (être musicien, s'exprimer) le rapport entre vêtement et musique se rapprocherait de la moyenne.

Une certaine tendance exhibitionniste serait alors typique des consommateurs-suiveurs, proches du passage à l'acte créateur (et, ici, proches mentalement des groupes de rock), de ceux qui, il y a 80 ans à la NouvelleOrléans, étaient nommés les "second lines" et suivaient en accompagnant quelquefois rythmiquement les parades de jazz (Billard, 1989, p. 18). Une certaine dépendance aux groupes et aux styles revendiqués ou cultivés se traduirait, donc, par un désir exhibitionniste de les marquer vestimentairement. Ce désir serait moins fort à la suite d'une certaine reconnaissance sociale de la personnalité "vraie". Certains indices statistiques trouvés par nous nous le feraient supposer mais il faudrait le vérifier plus systématiquement.

\section{V. - SYNTHÈSE ET CONCLUSION}

Généralement, le sexe est un élément essentiel de discours et de compréhension des phénomènes de contre-culture. Le rock est aussi, et surtout, une sortie d'énergie, composé de toute une ardeur dont les symboles sexuels ont fait scandale à leurs heures de gloire. L'adhésion au modèle rock est donc différente selon qu'on soit fille ou garçon.

En ce qui concerne le rapport entre vêtement et musique, on note une moins grande sensibilité affichée des garçons, même si les chiffres et les écarts par rapport à la moyenne sont peu importants entre les deux groupes.

Nous avons remarqué (Seca 1987, Boyer, Mignon, Daphy, 1986) que les garçons manifestent une plus grande expressivité dans l'affirmation, dérisoire et réaliste, de la réussite par l'argent, le sexe et d'une haute position sociale. A l'opposé, les filles voient la réussite sous la forme d'un bonheur avec les autres et dans un groupe genéralement familial (l'importance du contact humain). Plus spécifiquement, les lycéennes se disent plus prédisposées envers les arts au sens large, le désir de faire changer la société et les voyages. Les lycéens, quant à eux, sont proportionnellement plus nombreux à plébisciter l'activité sportive (Seca, 1987).

Le modèle musical rock attire beaucoup plus de garçons (proportionnellement à la moyenne). De la même manière, les filles seront moins nombreuses à vouloir faire de la musique même si elles sont proportionnellement en aussi grand nombre à être musiciennes.

Le rock attire (et a attiré) plus les lycéens que les lycéennes. Il y a plus d'ex-membres de groupes de rock et plus de musiciens jouant un instrument rock chez les garçons que chez les filles interrogées par nous (voir aussi Seca, 1988).

Ceci ne signifie pas que les filles ont moins de vocations artistiques, bien au contraire (16).

II semble plutôt qu'avec le rock, nous soyons en face d'un modèle d'expression musicale fortement masculinisé et répondant à une tradition culturelle où l'exhibition sexuelle et la présence corporelle ont été symbolisées par les hommes plus que par les femmes. Quand on analyse les chiffres des raisons avancées pour ne pas vouloir faire du rock en groupe, on s'aperçoit que les lycéennes expriment essentiellement un désir d'en rester au rôle d'auditrices. Elles expliquent aussi qu'elles sont peu attirèes et passionnées par le rock. Les garçons, au contraire, avancent plus des raisons évoquant un sentiment d'impuissance (manque de don, trop grandes difficultés, autres passions) devant l'art musical.

Les choses évoluent vite uniquement dans le domaine des médias et du vedettariat où fleurissent depuis longtemps nombre de femmes. L'évolution des mœurs culturelles semble, elle, plus déterminèe par des traditions et les représentations sociales classiques $\mathrm{du}$ rôle de 
l'homme et de la femme. Les résultats que nous avons trouvés dans notre enquête rejoignent ceux analysés par Boyer, Delcaux et Bounoure concernant les pratiques culturelles des lycéens:

"Les activités et goûts des lycéens sont sensiblement différents selon qu'il s'agit de filles ou de garçons et reproduisent les normes les plus traditionnelles de la division sexuelle des rôles (...) " (Boyer, Delcaux, Bounoure, 1987, p. 61).

La passion et les différentes formes par lesquelles elle s'exprime sont étroitement associées à des variables comme la sexualité et le sexe, l'éducation, la culture familiale, l'évolution individuelle. Les lycéennes, même si certaines d'entre elles affirment leur attrait pour le rock, expriment un peu à leur insue, que le rock a longtemps été une affaire d'hommes où les femmes tenaient, plutôt, un rôle de supporter chaleureux et "libéré".

Depuis le moment punk et son expression musicale asexuelle, les groupes de rock féminins (17) semblent avoir trouver un territoire symbolique qui leur conviennent plus, même si la violence rock et le décharnement stylistique punk les renvoient à une identité très fétichiste et urbaine.

Indépendamment de l'évolution de l'art rock, il apparaît, d'après nos enquêtes, que les hommes semblent plus soumis à son emprise.

Résumons, cependant, quelques points importants de l'analyse de l'audience du rock.

1. Le rock comme pratique est extrêmement minoritaire chez les lycéens et, a fortiori, chez les lycéennes que nous avons interrogés.

2. Le rock comme modèle diffus est assez influent $(35,2 \%$ des sujets connaissent personnellement un groupe de rock, $19,6 \%$ de l'ensemble, $25,6 \%$ des musiciens et $17,6 \%$ des non-musiciens désireraient appartenir à un groupe de rock).

3. Les lycéens sont essentiellement préoccupés $(80 \%)$ par leur avenir professionnel et universitaire même si une certaine ambivalence concernant leurs vocations existe. $58 \%$ disent avec précision quel type de profession ils désireraient exercer et quelles études ils veulent poursuivre; dans cette catégorie de lycéens $27,3 \%$ envisagent un avenir artistique. Ce qui est un chiffre appréciable.

4. L'influence du rock n'est pas traduisible en adhésion explicite a des courants stylistiques reconnus comme tels mais se concrétise par une intériorisation des valeurs musicales au sens large et par une extériorisation vestimentaire non-maîtrisée, dont la cohérence symbolique demeure approximative. Tout se déroule comme si les groupes de rock avaient une influence indirecte sur les choix esthétique des lycéens. Cette influence indirecte se traduirait par des activités rock après 18 ans. Cela correspond à une moyenne d'âge des musiciens rock qui tourne autour des 24-25 ans (Seca, 1988, p. 94). Ce qui ferait du rock une pratique d'accompagnement dans l'entrée dans la vie active et un espace artistique postadolescent.

5. L'audience des groupes de rock semble donc plus forte que l'examen des chiffres bruts ne le laisserait supposer. La prédominance d'un certain éclectisme musical et d'une relative tendance à l'affranchissement vis-à-vis des clichés véhiculés par les modes et la "culture-jeune " des médias va de pair avec une certaine indolence du teenager qu'est avant tout le lycéen (= machinerie idéale et idéalisée de la consommation culturelle de masse, ouverte donc malléable, adaptable à un marché en mouvement).

Peut-on, alors, parler d'une musicalisation de la société dont le rock formerait le noyau minoritaire mais à fort pouvoir d'attraction? II ne faut pas oublier que sept lycéens sur dix désirent faire de la musique ou pratiquent effectivement un instrument. L'attrait pour la musique est, selon nous, un indice important pour mesurer les recherches d'alternatives domestiques aux nécessités profondes d'une adaptation de l'individu aux structures sociales.

On est cependant Join, pour ces lycéens, de ce que Zofiropoulos et Pinell (1982) ont nommé le gauchisme culturel. On ne peut pas non plus " diagnostiquer" froidement qu'ils forment un ensemble amorphe et homogène de teenagers gavés par les marchandises culturelles.

La préoccupation majeure du lycéen semble être celle de sa vocation professionnelle et c'est paradoxalement le thème autour duquel les membres des minorités rock se mobilisent avec force et obstination (Seca, 1988). On peut, par conséquent, établir un lien important entre les deux termes de notre enquête à travers ce thème de la vocation.

Ajoutons enfin que le rock est formé de multiples représentations sociales qui entourent les sons et les styles autant que ceux-ci provoquent des transes en concert. C'est de ce rock imaginaire dont nous avons rendu compte.

Jean-Marie SECA Université de Reims 
(1) L'étude dont nous rendons compte, ici, s'est déroulée à Paris dans trois lycées (Lycée Voltaire, Lycée Colbert et Lycée Ravel). II s'agit d'une enquête par questionnaires auprès de jeunes de 15 à 18 ans suivant un cursus d'enseignement général, durant la période allant de janvier à février 1986. Nous remercions les Proviseurs de ces lycées pour leur aide.

(2) Patrick PARMENTIER a tenté, dans un tout autre domaine, de définir, avec une grande précaution et un soin particulier, des critères de classification des goûts des lecteurs de bibliothèques publiques. Celui-ci constate d'ailleurs: "la production même de l'objet a toujours à voir avec sa (ses) réception (s) possible (s), donc avec son existence sociale ultérieure et on s'aveugle toujours à une part de la réalité quand on autonomise l'objet culturel comme un pur produit expressif " (1986, p. 202).

(3) Que ce soit chez Dubet (1987), chez Tanner (1981), Murdock (1972) pour l'analyse du rock comme symbole esthétique d'un mode de vie "mauvais garçon ".

- que ce soit chez Parsons (1955), Maurice (1978), Conan (1979) pour marquer les activités du groupe " jeunes " d'un sceau d'insignifiance (période de transition selon Parsons) ou d'exaltation, d'éloge de son authenticité (Maurice, Conan).

- ou chez Erikson (1972) dans ses injonctions morales sur l'a éthique d'adulte " que tout un chacun, tôt ou tard est porté à assumer, les productions culturelles de l'adolescence sont marquées d'une limite et neutralisées comme mutilées, en crise, sans autonomie a réelle", etc.

(4) Chapman et Williams (1976) ont montré l'importance du contexte, du prestige d'une oauvre, donc du social sur l'écoute et la perception musicale. Rouget (1981) dans une approche plus anthropologique synthétisait, il y a quelques années, les analyses des relations existant entre mythes, représentations sociales et culturelles du corps et du plaisir musical.

(5) II est difficile, en effet, de distinguer entre les fonctions symboliques ou expressives d'une musique et l'attribution d'un trait négatif selon qu'on estime qu'elle est aimée par un groupe déviant (négativement apprécié) et un groupe majoritaire d'appartenance (valorisée positivement). La constitution d'un goût pour la musique obéit à des phénomènes d'influence très subtiles. AEBISCHER, HEWSTONE et HENDERSON (1984) ont montré d'une facon assez originale comment une musique minoritairement appréciée (le new-wave) chez des collégiens préférant le hard-rock était rejetée d'autant plus que les sujets étaient perméables à elle et informés que des jeunes élèves de LEP (bas statut) adoraient le new-wave. Les élèves rejetant le plus le new-wave étajent ceux qui auraient été perméables à elle. Ceux-ci choisissaient la musique contemporary considérée par les expérimentateurs comme proche de la new-wave pour se démarquer des élèves de LEP ayant mauvaise réputation dans les lycées.

Une preférence trop explicite pour la new-wave était traduite en goût plus prononcé pour la musique contemporary. Les lycéens gardaient un peu de l'esprit de la new-wave tout en se differenciant d'elle. Concernant l'importance des phénomènes d'influence indirecte, voir aussi Moscovici, Mugny (1988).
(6) Boyer (1988), Boyer, Delcaux et Bounoure (1987), Mignon, Daphy et Boyer (1986) ont, récemment, estimé, dans l'analyse des résultats de leur enquête en milieu lycéen, que les styles musicaux n'avaient pas une unité et une cohérence aussi stricte. Ce qui, implicitement, remet en cause, une fois de plus, la vision d'une "culture-jeunes" homogène dans ses références et ses modes d'appartenance sociale: "A l'image du sens commun d'une jeunesse unitaire se substitue le constat de la pluralité " (Boyer, Delcaux, Bounoure, 1987, p. 60).

(7) 51 lycéens $(18,1 \%)$ en connaissent un personnellement et 48 $(17,1 \%)$ un seul.

(8) Nous ne pouvons présenter, dans ce cadre, tous les tableaux que nous avons analysés. Le lecteur plus exigeant statistiquement pourra se référer à notre thèse (Seca, 1987). Le critère adopté ici est la différence par rapport à la moyenne alliée avec des recoupements de résultats d'analyses de correspondances.

(9) Alors que la moyenne est de $73,3 \%$ pour ceux qui fréquentent les concerts.

(10) Le fait de connaitre un ou plusieurs groupe (s) de rock a une moins grande influence sur le fait de vouloir faire de la musique. Ceux qui connaissent un ou plusieurs groupe (s) sont $63,5 \%$ a vouloir faire de la musique alors que ceux qui n'en connaissent pas sont $56.5 \%$. L'écart entre les deux groupes de lycéens est moins grana $(7 \%)$ que lorsqu'il s'agit de savoir s'il y a désir d'appartenance à un groupe de rock (écart de $18,9 \%$ ). On constate cependant qu'il y a quand même une plus grande volonté de faire de la musique chez ceux qui connaissent personnellement des groupes de rock.

(11) Ils sont $56,3 \%$ à voujoir appartenir à un groupe de rock alors que ceux qui n'ont pas fait partie de groupe par le passé ne sont que $17,8 \%$ à désirer une telle chose (la moyenne de réponses positives à la question du désir d'appartenance à un groupe de rock est $19,6 \%$ de l'ensemble.

(12) Les éclectiques sont définis par nous comme des amateurs rock ouverts à beaucoup d'influences stylistiques (jazz, classique, Edith Piaf, Brassens, Avant-garde, Musiques Andines, etc.).

(13) Les évaluations étaient faites en fonction d'échelles bi-polaires allant de -3 (pas du tout d'accord) à +3 (tout à fait d'accord) recodées de $1(=-3)$ à $6(=+3)$.

(14) Voir Seca (1987, annexes) à ce sujet pour plus de détails.

(15) De même, plus les groupes de rock sont considérés comme proches de la manière de vivre des lycéens et comme faisant changer le monde, plus le rapport estimé entre vêtement et musique écoutée, est grand (Seca, 1987).

(16) Dans notre enquête, elles sont trois fois plus nombreuses à affirmer une vision de leur avenir en termes d'activité artistique. C'est ce qui tes distingue d'ailleurs des garçons qui, sur tous les autres plans, semblent partager les mềmes visions de l'avenir qu'elles.

(17) II s'agit, cependant, là aussi de minorités. 
AEBISCHER V., HEWSTONE M., HENDERSON M., (1984). - Minority influence and musical preference. Innovation by conversion not coercion. Journal of European and Social Psychology, vol. 14, $\mathrm{n}^{\circ} 1,23-25$.

ALTHEIDE D.L., (1979). - The mass media and youth culture; Urban Education, vol. 14, $\mathrm{n}^{\circ} 2$, July.

BILLARD F., (1989). - La vie quotidienne des jazzmen américains jusqu'aux années 50, Paris Hachette.

BOYER R., (1988). - Les gouts musicaux des lycéens, in : Cahiers " Jeunesses et Sociétés ", $n^{\circ} 10$, février, 33-47.

BOYER R., DELCAUX M., BOUNOURE A., (1987). - Cultures Lycéennes. Revue Française de Pédagogie, $n^{\circ} 81$, oct., nov. déc., 55-62.

BOURDIEU P., (1980). - Questions de Sociologie. Paris, Editions de Minuit.

BUXTON D., (1985). - Le star système et la société de consommation. Ed. La Pensée Sauvage, Grenoble.

CHAPMAN A.J., WILLIAMS A.R., (1976). - Prestige effects and aesthetic experiences: adolescent's reaction to music (enquête lycée) ; British J. Soc. Clin. Psychol. 15, $n^{\circ}$ 1, 61-72.

CODOL J.P., (1979). - Semblables et différents. Thèse de Doctorat d'État. Université de Provence, UFR de Psychologie.

CONAN E., (1979). - Une génération sur la défensive, Rev. Esprit, 33-34.

DAPHY E., (1988). - Le groupe rock: rite de passage ou rite d'initiation?, in: Cahiers " Jeunesses et Sociétés, $\mathrm{n}^{\circ} 10$, tévrier, $71-78$.

DUBET F, (1987). - La Galère: jeunes en survie, Paris, Ed. Fayard.

ERIKSON E.H., (1972). - Adolescence et crise, Flammarion, Paris.

GALLAND O., (1984). - Les jeunes, Paris, Editions de la Découverte.

GERBOD P., (1988). - La musique populaire en France dans la deuxième moitié du $X X^{\ominus}$ siècle. Revue Ethnologie Française, XVIII, 1, janiv. mars, 15-26.

GREEN A.M., (1988). - Les conduites musicales des adolescents: Rêve d'une culture ou culture d'un rêve ? In: Cahiers “ Jeunesses et Sociétés", $n^{\circ} 10$, février, 57-70.

HENNION E., (1983). - Une sociologie de l'intermédiaire : le cas du directeur artistique de variétés, Sociologie du Travail, 4.83 , 459-473.

HENNION E., (1980). - Les Professionnels du disque, Paris, Ed. A.M. Métaillé.

HENNION A., VIGNOLLE J.P., (1978), - Les industries culturelles. L'économie du disque en France. DGRST. Paris, La Documentation Française.

HERSENT J.F., (1988). - Rock, jeunesse, socialisation. in : Cahiers "Jeunesses et Sociétés", $n^{\circ} 10$, février $48-56$.

INCIYAN E., (1987). - Au pays de la culture rock, in : Le Monde de l'Education, $n^{\circ} 144$, décembre 1987, 12-17.

JOHNSTONE J., KATZ E., (1957), - Youth and popular music: a study in the sociology of taste, The American Sociological Review, vol. LXII, May, $\mathrm{n}^{\circ} 6$.

LE GUIDE DE L'ÉYUDIANT, (1978). - Enquête chez les lycéens, in : Le Monde de l'Éducation, octobre 1978.

LEMAINE G., (1974). - Social Differentation and Social Originality, European Joumal of Social Psychology, 4, 17-52.
MAURICE F., (1978). - Le rock: une musique pour le présent, Rev. Esprit, n 22.

MC LUHAN M., (1965). - Pour comprendre les médias, Seuil, Paris.

MC LUHAN M., (1977). - D'ceil à oreille, Denoël Gonthier, Paris.

MIGNON P., (1988). - La production sociale du rock, in : Cahiers " Jeunesses et Sociétés ", $n^{\circ} 10$, février. 3-32.

MIGNON P., DAPHY E., BOYER R., (1986). - Les lycéens et la musique. INRP, collection "Rapport de Recherches" 1986 , $\mathrm{n}^{\circ} 2$.

Ministere de la Culture, (1982). - Les pratiques culturelles des Français. Paris, Ed. Dalloz.

MOSCOVICi S., (1976) (Nouv. éd.). - La Psychanalyse, son image, son public. Paris, Presses Univ. de Fr.

MOSCOVICl S., MUGNY G., (1988). - Psychologie de la Conversion, Fribourg, Delval.

MORIN E., (1966). - Adolescents en transition, Revue Française de Sociologie, VII, 435-455.

MURDOCK G., (1972). - Youth Culture and The School revisited, The British J. of Sociology, vol. XXIII, $\mathrm{n}^{\circ} 4,478-482$.

OBALK H., SORAL A., PASCHE A., (1984). - Les Mouvements de Mode expliqués aux Parents. Paris, Robert Laffont, Le Livre de Poche.

PARMENTIER P., (1986). - Bon ou mauvais genre: la classification des lectures et le classement des lecteurs. Bulletin des Bibliotheques de France, tome $31, n^{\circ} 3$, p. 203-223.

PARSONS T., (1955). - Age et sexe dans la société américaine, dans : Éléments pour une sociologie de l'action, Paris, Plon.

PERRIAULT J., BOFFETY B., BOUDINOT J.F., DAPHY ET AL., (1985). - Rock ou micro-informatique. Enquête sur des adolescents du $13^{\circ}$ arrondissement de Paris. INRP, collection "Rapport de Recherches", 1985, n 1 .

ROUGET G., (1981). - La musique et la transe, Gallimard, Paris.

SECA J.M., (1991). - Les purificateurs rock (ronéo) (en cours de publication).

SECA J.M., (1988). - Vocations Rock. L'État Acide et l'Esprit des Minorités Rock. Paris, Méridiens Klincksieck Editeur, collection "Psychologie Sociale".

SECA J.M. (1987). - L'état acide: analyse psycho-sociale des minorités rock, Thèse de Doctorat, Université de Paris $X-$ Nanterre.

SOMMER C.M., WIND P., (1986). - Menschen, Stile und Kreationen, Frankfurt, Berlin, Ullstein Sachbuch, Kol. «Popular Kultur".

TAJFEL H., (1978). - Differenciation between social groups: the social psychology of intergroup relations. London, Academic Press.

TANNER J., (1981). - Pop music and peers groups: a Study of Canadian High School Student's responses to pop music, Rev. Can. Sociol. Anthropol. 18, $n^{\circ} 1$.

VIGNOLLE J.P. (1980). - Mélange des genres alchimie sociale, la production des disques de variétés. Sociologie du Travall, 22 $n^{\circ} 2,129-151$.

YONNET P., (1985), Jeux, Modes et Masses. Paris, Gallimard.

ZOFIROPOULOS M., PINELL P., (1982). - Drogue, Déclassement et Stratégie de Déqualification. Actes de la Recherche en Sciences Sociales, 42, 61-75. 